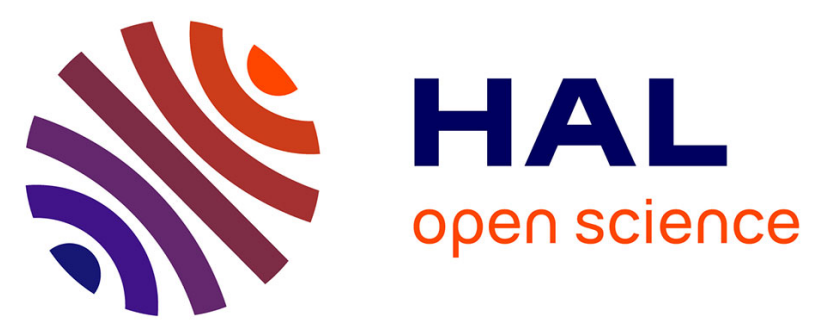

\title{
Viscoelastic and dielectric properties of 5CB nematic liquid crystal doped by magnetic and nonmagnetic nanoparticles
}

Habib Ayeb, Mouna Derbali, Ahmed Mouhli, Taoufik Soltani, Fathi Jomni, Jérôme Fresnais, Emmanuelle Lacaze

\section{To cite this version:}

Habib Ayeb, Mouna Derbali, Ahmed Mouhli, Taoufik Soltani, Fathi Jomni, et al.. Viscoelastic and dielectric properties of 5CB nematic liquid crystal doped by magnetic and nonmagnetic nanoparticles. Physical Review E , 2020, 102 (5), 10.1103/PhysRevE.102.052703 . hal-03070728

\section{HAL Id: hal-03070728 https://hal.science/hal-03070728}

Submitted on 15 Dec 2020

HAL is a multi-disciplinary open access archive for the deposit and dissemination of scientific research documents, whether they are published or not. The documents may come from teaching and research institutions in France or abroad, or from public or private research centers.
L'archive ouverte pluridisciplinaire HAL, est destinée au dépôt et à la diffusion de documents scientifiques de niveau recherche, publiés ou non, émanant des établissements d'enseignement et de recherche français ou étrangers, des laboratoires publics ou privés. 


\title{
Viscoelastic and dielectric properties of $5 \mathrm{CB}$ nematic liquid crystal doped by magnetic and nonmagnetic nanoparticles
}

\author{
Habib Ayeb*, Mouna Derbali, Ahmed Mouhli, and Taoufik Soltani \\ Université de Tunis El Manar, Faculté des Sciences de Tunis, LR99ES16 Laboratoire \\ de Physique de la Matière Molle et de la Modélisation Electromagnétique, 2092 Tunis, \\ Tunisie \\ Fathi Jomni \\ Université de Tunis El Manar, Laboratoire Matériaux Organisation et Propriétés \\ (LR99ES17), 2092, Tunis, Tunisie \\ Jérôme Fresnais \\ Sorbonne Universités, UPMC Univ Paris 06, UMR 8234, PHENIX, F75005 Paris, \\ France \\ Emmanuelle Lacaze* \\ Sorbonne Universités, UPMC Univ Paris 06, CNRS UMR 7588, Institut des Nano- \\ Sciences de Paris (INSP), F75005 Paris, France
}

In this article we show how spherical nanoparticles (NPs) imposing planar anchoring can strongly impact the viscoelastic, dielectric and electro optical properties of a nematic liquid crystal when they are not aggregated. We also demonstrate that when the NPs are magnetic most nematic properties are more impacted than when they are non-magnetic. With magnetic NPs a molecular disorder is induced that decreases the nematic order parameter, this decrease impacting the values of elastic constants, viscosity and response time. The impact on 5CB liquid crystal (LC) has been investigated with spherical nanoparticles (NPs) of identical size around $6 \mathrm{~nm}$, magnetic $\left(\gamma \mathrm{Fe}_{2} \mathrm{O}_{3}\right)$ and nonmagnetic $\left(\mathrm{CeO}_{2}\right)$ ones, that are both surface functionalized by Poly(amninopropylmethylsiloxane-b-dimethylsiloxane) (PAPMS-b-PDMS) block copolymer ligands to promote planar anchoring. In presence of non-magnetic NPs, despite an almost constant nematic order parameter, a significant decrease of elastic constants $(25.4 \%)$, viscosity (22\%) and response time (23\%) is measured. It suggests a dilution effect for the intermolecular interactions in presence of NPs. This hypothesis is supported by the observation of an enhanced decrease of the same nematic parameters in presence of magnetic NPs that can be fully explained by the corresponding order parameter decrease. This finally leads to a remarkable decrease of the splay elastic constant by $51 \%$ in presence of magnetic NPs. The decrease of the nematic order parameter by $18 \%$ in presence of magnetic NPs demonstrates that the NP magnetic moments are only weakly coupled to the nematic director and consequently only induce a disorder in the composite system. A significant influence of the expected large LC structural modifications in presence of magnetic NPs is however shown by a particularly large increase of the diffusion coefficient (43\%) and large decrease of the dielectric anisotropy (43\%). We believe that the observed impact of NPs with planar anchoring on nematic properties could be extended to most spherical NPs if their aggregation can be avoided. In particular the difference between magnetic and nonmagnetic NPs could be extended to ferroelectric and non-ferroelectric NPs. 
Keywords: Nematic liquid crystal; $\gamma \mathrm{Fe}_{2} \mathrm{O}_{3} ; \mathrm{CeO}_{2}$; Electro optical response;

Dielectric anisotropy

DOI:

*Corresponding authors: emmanuelle.lacaze@insp.jussieu.fr; ayebhabib@gmail.com

\section{INTRODUCTION}

Nematic liquid crystals (LCs) are anisotropic fluids, easily distorted by external electric or magnetic fields, this characteristic making them widely used in display technologies (LCDs). However, the performance of the display device is mainly associated with a weak driving voltage and a fast response time. During the last two decades the research field of liquid crystals and their classical applications are undergoing changes towards new and diverse applications in the area of nanoscience and nanotechnology. This new research field has been strongly stimulated by experiments of nanoparticle doping in order to obtain new LC characteristics such as dielectric anisotropy enhancement, faster switching time, and low driving voltage.

A lot of NP/LC composite were studied including metallic NPs [1-6], semiconducting NPs [7-10], carbon nano-tubes [11,12], insulating [13-14] NPs and quantum dots [15-19]. However, a lot of contradictory results have been obtained, in particular regarding the evolution of the elastic constants in presence of NPs, responsible for most interesting LC characteristics. Different behavior for $K_{1}, K_{2}$ and $K_{3}$ have been observed sometimes with the same behavior for $K_{1}, K_{2}$ and $K_{3}$ [20-22], sometimes not [23,24], sometimes increasing [25,26], sometimes decreasing [27,28]. As a result, other physical parameters are accordingly affected like dielectric and viscoelastic properties [23,25,29].

One difficulty is that NPs of different nature are used, but also of different shape, size and nature of the ligands [22,30,31]. This latter parameter may be a key parameter because the nature of the ligands usually determines the nature of the LC anchoring on the surface of the NPs. Recent work has compared the role of two particles of different natures, namely iron oxide $\left(\gamma \mathrm{Fe}_{2} \mathrm{O}_{3}\right)$ and cerium oxide $\left(\mathrm{CeO}_{2}\right)$, one of which being magnetic $\left(\gamma \mathrm{Fe}_{2} \mathrm{O}_{3}\right)$, the two types however displaying identical shapes, sizes and ligands [4]. It was shown that the magnetic threshold $\left(H_{C}\right)$ and the diffusion constant $(D)$ are both strongly affected by the presence of NPs but behaved differently depending on the type of NPs. This allowed establishing two different types of influence for the NPs: the one of inactive NPs (nonmagnetic $\mathrm{CeO}_{2}$ with PAPMS-b-PDMS block copolymer ligands) which imposes a weak planar anchoring; and the one of magnetic NPs (magnetic $\gamma \mathrm{Fe}_{2} \mathrm{O}_{3}$ with PAPMS-b-PDMS block copolymer ligands). These latter ones can be considered, as long as no magnetic field is imposed, as NPs which present a long-range interaction with the LC, thus leading to a strong planar anchoring parallel to the magnetic moment of the NPs. However, too few parameters have been studied to confirm this interpretation. We consequently propose in the present work to take up these two types of NPs and to extend their study to more LC parameters: the order parameter, dielectric anisotropy, rotational viscosity, electric threshold voltage, response time, splay and twist elastic constants. We find a different evolution of the order parameters for the two kinds of NPs. We observe no variation for non-magnetic NPs in relation with the weak planar anchoring on NPs. In contrast, we observe a net decrease of the order parameter for the magnetic NPs in relation with the disorder of the magnetic moment which drives the orientation of the planar molecules anchored on the spherical NPs. In this latter case, the NP magnetic moment appears to be not enough coupled with the nematic molecules to allow for a significant orientation of the NP 
magnetic moment, in contrast with a ferronematics made with large magnetic platelets [32-35]. We show that this different evolution of the order parameters is responsible for the different behaviors of the elastic constants and viscosity. However the observed decreasing of elastic constants and viscosity for non-magnetic NPs with non-varying order parameter also lead to the demonstration of a facilitation of molecular LC motions in presence of NPs most probably due to some dilution features in presence of NPs weakening in average inter-molecular interactions.

\section{EXPERIMENTAL}

\section{A. Materials and samples}

In this work, we used the well-known 5CB (4-pentyl-4-biphenylcarbonitrile) nematic liquid crystal purchased from Merck. The 5CB liquid crystal is in the nematic phase for temperatures ranging from 22 to $35.1^{\circ} \mathrm{C}$ with a high positive dielectric anisotropy.

Mixtures of $5 \mathrm{CB}$ doped with magnetic $\left(\gamma \mathrm{Fe}_{2} \mathrm{O}_{3}\right)$ and nonmagnetic $\left(\mathrm{CeO}_{2}\right)$ nanoparticles (NPs) were used in this study. Both kinds of NPs were spherical shaped with an average diameter $6.2 \mathrm{~nm}$ and $6.6 \mathrm{~nm}$ respectively and were synthesized by $\mathrm{J}$. Fresnais using a procedure described in reference [4]. This led to almost identical spherical NPs of similar shape, size and ligands (PAPMS-b-PDMS block copolymer ligands), one being non-magnetic $\left(\mathrm{CeO}_{2}\right)$, the other one being magnetic $\left(\gamma \mathrm{Fe}_{2} \mathrm{O}_{3}\right)$. We have varied the NPs concentration (weight fraction) in the $5 \mathrm{CB}$ nematic from 0 to $10 w t \%$. The pure $5 \mathrm{CB}$ or the $5 \mathrm{CB} / \mathrm{NPs}$ mixtures were filled into an empty cell purchased from (E.H.C. Japan) by capillary action in the isotropic phase. Each empty cell consists of two parallel glass slides, which surfaces are covered with a layer of unidirectionally rubbed (polyimide) to ensure uniform planar anchoring of the 5CB nematic. Each inner face of the slides was covered by a thin ITO layer to obtain electrodes with an active area of $25 \mathrm{~mm}^{2}$. All empty cells have thickness of $3.2 \pm$ $0.1 \mu \mathrm{m}$. To perform our measurements, the cell is placed into a homemade temperaturecontroller with an accuracy of $\pm 0.01^{\circ} \mathrm{C}$.

\section{B. Dielectric measurements}

The dielectric measurements of pure $5 \mathrm{CB}$ and the $5 \mathrm{CB} / \mathrm{NPs}$ mixtures were carried out using the impedance/gain phase analyzer (Solartron SI 1260) coupled to 1296 dielectric interface. The dielectric permittivity of the samples was measured in the frequency range from $100 \mathrm{~Hz}$ to $2 \mathrm{MHz}$ and the samples temperature was controlled by means of a Linkam TMS 94 controller with an accuracy of $\pm 0.1^{\circ} \mathrm{C}$. The perpendicular component of the relative permittivity $\left(\varepsilon_{\perp}\right)$ was measured using a planar aligned cell without application of an external electric field. However, we applied a DC voltage of 10 Volts to the planar cell to achieve homeotropic alignment, in order to measure the parallel component of the dielectric permittivity $\left(\varepsilon_{\|}\right)$.

To test the performance of our experimental setup, we performed the measurement of the dielectric constant of the pure 5CB liquid crystal at different temperatures. We obtained a good agreement with results carried out in previous works [36-38] in about 3\%. At constant temperature of $27^{\circ} \mathrm{C}$ we found $\Delta \varepsilon(5 C B)=12.10$, which is very close to 12.50 found by the authors of reference [36], 12.00 obtained by [37] and 12.75 measured by the authors of reference [38]. 


\section{Electro optical measurements}

To study the electro-optic properties of our samples (pure 5CB and doped one) an electric field was applied to the filled cell, which causes the molecules of liquid crystal to alter their orientations and change their optical properties. The cell is placed between crossed polarizers under polarizing microscope brand Microscope Eclipse LV100N Nikon. The sample is connected to an Agilent 33220A function waveform generator which can provide positive electric pulses or sinusoidal signal. We perform synchronous acquisitions of the electric current across the cell during the application of the electric field, the electric current data being acquired by a PC connected via a GPIB interface. The cell was connected in series to an external electric resistance $1 k \Omega$. We measure the current from the voltage across the resistor which is detected by means of a digital oscilloscope (Agilent infiniium DSO 5052A 500MHz, 4GSa/s). Typical transient currents across cells filled by pure $5 \mathrm{CB}$ and $5 \mathrm{CB}$ doped with $5 \%$ of magnetic or nonmagnetic NPs are shown on Fig. 1. The cell is subjected to a DC voltage for 1 second, with a magnitude $V=14$ Volts greater than the threshold $\mathrm{V}_{t h}$, at fixed temperature. All measurements were performed at $27^{\circ} \mathrm{C}$.

The reorientation of nematic molecules can be electrically observed as a realignment of the nematic director from a perpendicular to a parallel state with respect to the applied electric field, which results in the appearance of an important peak in the electric transient current across each cell as we can see on Fig. 1. The nematic director rotation is characterized by a response time given by the following formula [39]:

$$
\tau=2 \gamma_{1} /\left(\varepsilon_{0} \Delta \varepsilon E^{2}\right)
$$

Where $\gamma_{1}$ is the rotational viscosity, $E=V / d$ the applied electric field, $d$ the cell thickness, $\Delta \varepsilon$ is the dielectric anisotropy and $\varepsilon_{0}$ is the permittivity of the vacuum. Experimentally the response time corresponds to the time measured between the instant at which the electric field is applied and the maximum of the current peak.

\section{RESULTS}

Figure 2 shows the behavior of the dielectric anisotropy of pure nematic 5CB and 5CB-doped with magnetic (red circle) and nonmagnetic (blue square) NPs measured at frequency $10 \mathrm{kHz}$ and constant temperature of $27^{\circ} \mathrm{C}$. The value of the dielectric anisotropy decreases on increasing the concentration from 0 to $5 \mathrm{wt} \%$ for both kinds of NPs. By varying the concentration from 0 to $5 \%, \Delta \varepsilon$ decreases by $16 \%$ for nonmagnetic NPs and $43 \%$ for magnetic NPs at the same temperature conditions of reference [4]. For concentration larger than 5\%, the dielectric anisotropy starts to increase with NP concentration.

Figure 3 presents the evolution of the response time $\tau$ as a function of the nanoparticle weight fraction concentration for nonmagnetic (blue square) and magnetic nanoparticles (red circle). Figure 3 shows that for both NPs, the response time decreases from its initial value (for pure $5 \mathrm{CB}$ ) to reach a minimum at a concentration equal to $5 \%$ but the gap between the two kinds of particles remains small (about 23\% of decrease for nonmagnetic NPs against 20\% for magnetic NPs).

Figure 4 shows the variation of the rotational viscosity $\gamma_{1}$ as a function of the NP concentration for nonmagnetic (blue square) and magnetic NPs (red circle). The rotational viscosity $\gamma_{1}$ was determined by fitting the experimental data of the response time $\tau$ versus the applied electric field using the above equation (1). The obtained value of $\gamma_{1}$ for the pure 5CB at the working temperature is in good agreement with many previous results [40-43]. The general behavior of the rotational viscosity $\gamma_{1}$ curve appears to be similar to the response time $\tau$ and the dielectric anisotropy $\Delta \varepsilon$, in other 
words, $\gamma_{1}$ decreases (by about 22\% and 37\% respectively for nonmagnetic and magnetic NPs) with the concentration to reach a minimum at $c=5 \%$ and then increases by increasing the concentration.

Figure 5 shows the behavior of the threshold voltage of cells filled with pure 5CB and $5 \mathrm{CB}$ doped with magnetic and nonmagnetic NPs versus concentration. The results show a threshold decrease until $c=5 \%$ followed by slight increase but without reaching back the initial value. The threshold voltage decreases down to $5.9 \%$ and $7 \%$ respectively for 5CB doped with nonmagnetic and magnetic NPs.

Figure 6 shows the variation of the splay $K_{1}$ and twist $K_{2}$ elastic constants as a function of the NPs concentration for nonmagnetic (blue square) and magnetic NPs (red circle). The twist elastic constant $K_{2}$ was deduced from the experimental data of the diffusion constant $D=K_{2} / \gamma_{1}$ presented in reference [4] while $K_{1}$ is directly related to the threshold voltage by the following equation:

$$
K_{1}=\left(\mathrm{V}_{t h} / \pi\right)^{2} \varepsilon_{0} \Delta \varepsilon
$$

Where $\Delta \varepsilon$ and $V_{t h}$ are respectively the experimental measured dielectric anisotropy and threshold voltage. The obtained values of $K_{1}$ and $K_{2}$ of the pure $5 \mathrm{CB}$ at the working temperature are in good agreement with previous results [44], being smaller but still close to the value for elastic constants reported in other papers $[36,38,40]$. From figure 6 one observes a remarkable decrease of $K_{1}$ (of about 51\% and 25.4\% respectively for magnetic and nonmagnetic NPs) for both kinds of NPs. The decrease of $K_{2}$ is smaller. This smaller decrease may be mostly related to a non-monotonic behavior with an initial increasing for the concentration between 0 and $3.2 \%$.

\section{DISCUSSION}

The first observation that we can highlight is that all physical parameters show the same concentration dependency with two concentration regimes: at low concentration quasi all physical parameters show a significant decrease to reach a minimum at $c=5 \%$. Then an increase is observed at larger NP concentration. This is the same dependency than the one found in reference [4] where the low $c$ regime has been interpreted as related to a dominating influence of single NPs whereas the large $c$ regime may be related to dominating aggregates. $c=5 \%$ weight fraction corresponds to $3.5 \times 10^{-2}$ volume fraction as aggregation limit. This limit is high compared to many previous works where the limit is mostly found around $10^{-4}[29,30,45-47]$. Aggregation occurring for a large NP volume fraction has been related to the functionalization with the polydimethylsiloxane (PDMS) [4], and to the corresponding weak planar anchoring in contrast with ligands inducing homeotropic anchoring $[22,30,31]$. These findings point out that, in order that NPs significantly affect the nematic properties, the NP concentration could not exceed the $c=5 \%$ limit. Indeed, the presence of aggregates in the nematic matrix reduces the effect of the NPs on LC properties.

The second observation is that the origin of the phenomena induced by both kinds of NPs needs to be differently interpreted. The decrease of most parameters is indeed different despite the fact that they are very close in size, shape and functionalization. This confirms that the two behaviors are related to the different nature of the NPs, as discussed below.

\section{A. Nonmagnetic nanoparticles}

Let us first analyze the effect of the nonmagnetic NPs on the nematic matrix with a focus on the small concentration regime where the influence of isolated particles dominates. 
In order to determine the evolution of the order parameter $S$, as a function of the NP concentration, we have measured the variation of $\Delta \varepsilon$ as a function of the temperature in absence of NP. We have also measured the evolution of the nematic isotropic phase transition temperature $T_{N I}$ as a function of the NP concentration and the results are listed in Table 1. We use the Haller model [48] showing that $S(T)=$ $\Delta \varepsilon(T) / \Delta \varepsilon(0)$ and the fact that $\Delta \varepsilon(T)=A\left(T_{N I}-T\right)^{\beta}$, which leads to :

$$
S=\left(1-\frac{T}{T_{N I}}\right)^{\beta}
$$

For the determination of $S$ as a function of the NP concentration, we assume that the variation of $\beta$ with the NP concentration can be neglected. This is consistent with the demonstration by Kundu et al. [49] that for various mixtures of calamitic and bent-core materials the value of $\beta$ remains constant, very close to the value that we have obtained for pure $5 \mathrm{CB}$ through the fitting of the measured curve of $\Delta \varepsilon(T): \beta \approx 0.18 \pm 0.01$, a value also in good agreement with measurements of many other works [48,50,51]. For pure $5 \mathrm{CB}$, we measured $T_{N I}(5 C B)=35.1^{\circ} \mathrm{C}$ and we found $S=0.538$ which is very close to the value measured by others [40,52].

The results for the $S$ variation in relation with the measured $T_{N I}$ variation are shown in figure 7 . The small decrease of $T_{N I}$ by about $1.5^{\circ} \mathrm{C}$ in average remains out of the error bar estimated at $\pm 0.1^{\circ} \mathrm{C}$ but reveals an only small $S$ variation down to a maximum decrease of $3 \%$. This result reinforces the approximation that was taken in our previous work [4] that $S$ remains mostly constant when the NP concentration is increased. This absence of significant variation for the order parameter may be mainly related to the weak planar anchoring induced by the ligands that allow for an easy adaptation of the 5CB molecules anchored on the NPs to the surrounding nematic LC (see Figure 8a).

If $S$, the order parameter, varies only few with the NP concentration, in contrast the elastic constants vary a lot. Figure 6 shows the behavior of $K_{1}$ and $K_{2}$, respectively splay and twist elastic constants. $K_{1}$ significantly decreases by $25.4 \%$ to reach a minimum at $5 \%$ of NP concentration. This behavior may be similar to an also large decrease of $K_{3}$ that may be responsible for the previously observed large decrease of the magnetic threshold in presence of the same NPs [4]. The behavior of $K_{2}$ is not so clear. A decrease is indeed found around $c=5 \%$ weight fraction, but for smaller NP concentration a slight increase is observed. It is well known that $K_{1}$ is proportional to $S^{2}$ [44,51]. We can write $K_{1}$ as following: $K_{1}=f(c) S^{2}$ (similarly for $K_{2}$ and $K_{3}$ ) where $f(c)$, a priori dependent on the NP concentration, defines the proportionality of $K_{1}$ to the scalar order parameter $S$. Since $S$ is concentration independent, $f(c)$ must strongly decrease with the NP concentration down to a decrease by a factor of 0.79 for $c=5 \%$. This behavior may be related to the one of the rotational viscosity, decreasing by $22 \%$ for $c=5 \%$ weight fraction. With a weak planar anchoring, if we believe that the NPs could rotate when the electric field is applied, a NP rotation may facilitate the realignment of the nematic molecules to its direction and therefore the value of the rotational viscosity may be reduced. Another possibility is that due to the presence of NPs, a dilution occurs in the nematic material, leading to an average decrease of intermolecular interactions that may decrease the average viscosity [25,53]; A facilitation of molecular disorientation in relation either to easy NP rotation or to dilution of the intermolecular interactions would also decrease the splay elastic energy as observed.

The same feature should apply to bend and twist distortion. This may explain the strong decrease of the magnetic threshold in presence of these NPs [4]. A decrease of 
$K_{2}$ is also shown on Figure 6 but less clearly than for $K_{1}$. We believe that it is due to the fact that the $K_{2}$ behavior shown on Figure 6 has been obtained by the connection between two results, the $D$ evolution determination in [4] and the $\gamma_{1}$ one, both having been obtained with the same solution of NPs but one being just prepared [4] and the other one having been prepared more than one year before the measurement. We can thus anticipate that the density of ligands may have slightly changed before the measurements, thus varying the planar anchoring energy between the two measurements. As a result, even if the feature is visible around $c=5 \%$ weight fraction, the $K_{2}$ measurement may be not so accurate than the $K_{1}$ one. Finally, as shown by Figure 3 , the time constant also significantly decreases, the molecular rotation being facilitated by the NP presence a faster molecular rearrangement is induced.

In order to compare the present results to the results of the literature, it is important to compare to the measurements made with NPs, with neither magnetic nor ferroelectric property. They could indeed differently influence the LC, due to long range magnetic or electric interactions respectively. Our results appear to be similar to the ones obtained by Yadav et al. [54] with $\mathrm{SiO}_{2}$ NPs. However, they differ from a number of other measurements. This may be due to different shapes of NPs [55,56], but also, for a number of cases this may be due to different anchorings at the NP surface or to quick aggregation that could occur for other kinds of functionalization of NPs [22, ,30, 31,5759]. Katiyar et al. [28] investigated the impact of $\mathrm{TiO}_{2} \mathrm{NPs}$ in $1823 \mathrm{~A}$ nematic liquid crystal. They observed that most physical parameters (dielectric anisotropy, viscosity, elastic constant) firstly decrease with the concentration and increase again when the concentration further increases similarly to the above results. However, the threshold voltage increases continuously when the concentration of NPs increases which appears contradictory with the expected proportionality of $\mathrm{V}_{T h}$ with $K_{1}$ and $\Delta \varepsilon$.

Our results finally show that with non-magnetic NPs and obviously also with nonferroelectric NPs, planar anchoring only induces a small variation of the order parameter for nematic LC like 5CB. In contrast the elastic constants, rotational viscosity and response time under electric field vary largely more, decreasing for example by $22 \%$ for viscosity and by $25.4 \%$ for splay elastic constants. This demonstrates a facilitation of molecular rotation in presence of NPs, either due to an easy rotation of the NPs, or due to a dilution effect of intermolecular interactions in presence of NPs.

\section{B. Magnetic nanoparticles}

As underlined by all experimental results here presented, it is clear that the magnetic NPs affect the nematic properties more than the nonmagnetic ones. For magnetic NPs, by varying the concentration from 0 to $5 \%, K_{1}$ was reduced by $51 \%, \gamma_{1}$ decreased by $37 \%$ and $\Delta \varepsilon$ decreased by about $43 \%$.

Figure 7 shows that the magnetic NPs (red circle) induce a significant decrease of the orientational nematic order parameter by about $18 \%$ when the concentration varies from 0 to $5 \%$. This is in contrast with the assumption made in ref [4] and may be due to the NP magnetic dipolar moments being randomly oriented as shown on Figure 8b. This indicates that the nematic structure cannot orient the magnetic moments of these spherical NPs in the direction of the director. If the NP magnetic moment would have been oriented by the nematic structure $S$ would have been larger than with non-nematic NPs. On the contrary this is the disorder of the NP magnetic moment that increases the disorder of the liquid crystal in relation with the strong planar anchoring that NPs induce through the long range magnetic interactions between NPs and LC (Figure 8b). The coupling between the magnetic NPs and the surrounding LC material is thus weak, in contrast with ferronematics made with platelet magnetic NPs $[35,60]$, either due to 
the small size or to the spherical shape of the magnetic NPs. The origin of the observed increased disorder displayed by the $S$ decrease may thus be of different origin than the one observed by Haraguchi et al. [61] who showed a reduction ranging from 0 to $30 \%$ of the order parameter $S$ by doping the ZLI-4792 nematic by $\mathrm{MgO}$ and $\mathrm{SiO}_{2} \mathrm{NPs}$, of size 10 and $17 \mathrm{~nm}$ respectively in relation with an observed reduction of clearing temperature by $4-5^{\circ} \mathrm{C}$ of the doped nematic with respect to the undoped nematics. The reduction of the clearing temperature was attributed to the nature of ligand molecules covering the nanoparticles [61]. In this latter case the results may be mostly related to an anchoring close to be homeotropic with non-ferroelectric and nonmagnetic NPs. On the other hand Gdovinová et al. [62] observed that doping the nematic LC 6CHBT by magnetic nanorods increases its nematic isotropic transition temperature $T_{N I}$ whereas doping with spherical magnetic NPs decreases it in agreement with our results.

This decrease of $S$ however does not explain the observed reduction of $K_{1}$ and $\gamma_{1} . K_{1}$ decreases until $c=5 \%$, to about half its value for the pure nematic LC and increases for further increase of NP concentration. This is consistent with the observed decrease of $S$ but it could not be explained only by the order parameter $S$ variation since $K_{1}$ is proportional to $S^{2}$ and $S^{2}$ decreases only by a factor 0.67 . The proportional term $f(c)$ then again also decreases with the NP concentration. In order to obtain $K_{1}(5 \%) /$ $K_{1}(0)=0.49, f(5 \%) / f(0)$ must be equal to 0.73 . This value is similar to the one for non-magnetic NPs (0.79). This indicates that the facilitation of molecular orientation is similar with magnetic NPs and non-magnetic ones without any significant enhancement induce by the expected long-range magnetic interactions. The same observation holds for $\gamma_{1}$ since the decrease in presence of magnetic NPs with respect to non-magnetic NPs is more efficient by 0.81 , in agreement with $\gamma_{1}$ being proportional to $S$ [42, 63-67] and the larger $\gamma_{1}$ decrease being only monitored by the $S$ decrease. These results finally shed a new light on the previous results outlined in reference [4] for magnetic NPs. They showed that the magnetic threshold, $H_{C}$, with magnetic NPs decreased by $31 \%$ whereas it decreased by $23 \%$ with non-magnetic NPs. $H_{C}$ being expected to be proportional to square root of the order parameter [4], we obtain again that this large decrease of $H_{C}$ is mostly monitored by the order parameter decrease. This shows that the driving force for the decrease of $H_{C}$ is not the orientation of the magnetic NPs induced by the external magnetic field but mostly the small value of the order parameter in presence of magnetic NPs. This observation that long range magnetic interactions do not promote a further facilitation of molecular orientation suggests that the assumption relating the observed decrease of the elastic constants and viscosity to a dilution effect may be the more appropriate.

This latter interpretation is also consistent with the observed decrease of NP impact for all parameters once NP aggregates dominate. The number of molecules with reduced intermolecular interactions corresponds to the number of molecules close to the NP surface. The surface of $N$ assembled NPs in a spherical aggregate scale as $N^{2 / 3}$ instead of $N$ for the same number of isolated NPs indeed leading to a decrease of the dilution effect when aggregates form. Accordingly Podoliak et al. [25] showed for two types of liquid crystals (TL205 and 18523) doped by $\mathrm{BaTiO}_{3}$ and $\mathrm{Sn}_{2} \mathrm{P}_{2} \mathrm{~S}_{6}$ ferroelectric NPs a decrease of the elastic constant by $8.3 \%$ and 9.5\% for TL205 doped respectively by $\mathrm{BaTiO}_{3}$ and $\mathrm{Sn}_{2} \mathrm{P}_{2} \mathrm{~S}_{6}$ and a more important decrease was observed $(11.6 \%$ and 20.1\%) for 18523 doped by $\mathrm{BaTiO}_{3}$ and $\mathrm{Sn}_{2} \mathrm{P}_{2} \mathrm{~S}_{6}$ respectively. They related this decrease to a dilution of the nematic liquid crystal when the NPs are introduced but unexpectedly, they observe an associated increase of the rotational viscosity. 
We finally show that for spherical magnetic NPs of small size $(6 \mathrm{~nm})$, there is an only weak coupling with the nematic order parameter. As a result, the nematic order parameter is not able to orient the NP magnetic moments. Reversively the disordered NP magnetic moments without external magnetic field impose an enhanced disorder to the nematics through the strong planar anchoring around the NPs, mediated by the longrange magnetic interactions (Figure 8b). This decrease of nematic order parameter induces a larger decrease of the elastic constants, rotational viscosity and response time under electric field with respect to non-magnetic NPs. However, no further facilitation of molecular reorientation is observed despite the expected strong planar anchoring on the NPs. This suggests that the facilitation process is due to a dilution effect of intermolecular interactions in presence of NPs. The modified structure of the LC associated with the long range magnetic interactions (Figure 8b) appears to only specifically impact two parameters in addition to the order parameter: firstly the diffusion coefficient which has been shown to increase much more with magnetic NPs (increase of 43\%) than with non-magnetic NPs (increase of 18\%) [4] whereas $D$ is expected to be close to be proportional to the order parameter, $S$, being equal to $K_{2}$ over the viscosity $\gamma_{1}$; secondly $\Delta \varepsilon$, decreasing by $43 \%$ with magnetic NPs and by $16 \%$ with non-magnetic NPs whereas it is expected to be proportional to the order parameter. The relationship between the specific structure of LC in presence of magnetic NPs (Figure 8a) and the large evolution of the two parameters is not clear. However, the observed variation of $D$ and $\Delta \varepsilon$ at least confirms a significant modification of the LC structure in presence of magnetic NPs, as suggested by Figure 8b.

\section{CONCLUSION}

The impact of magnetic $\left(\gamma \mathrm{Fe}_{2} \mathrm{O}_{3}\right)$ and nonmagnetic $\left(\mathrm{CeO}_{2}\right)$ nanoparticles (NPs) of similar size (around $6 \mathrm{~nm}$ ), shape (spherical) and ligands promoting planar anchoring (PAPMS-b-PDMS block copolymer ligands), on viscoelastic, dielectric and electro optical properties of nematic 5CB was studied. Due to the fact that until a NP concentration of $5 \% w t$, the dominating regime is the one of isolated NPs, in contrast with the regime where aggregates dominate for NP concentration larger than $5 \% w t$, we can deeply explore the influence of isolated NPs. Our results shed light for the specific case of planar anchoring induced by NPs on the difference between inert NPs and active ones (magnetic NPs and also possibly ferroelectric NPs). They demonstrate that it resides mainly in order parameter variations that are observed for the latter NPs only. For the non-magnetic NPs, despite the non-varying order parameter in presence of NPs, most physical properties decrease (elastic constants by $25.4 \%$, rotational viscosity by $22 \%$, response time by $23 \%$ ). This can be interpreted as a dilution effect of the intermolecular interactions in presence of NPs. For magnetic NPs, this dilution effect, combined with a significant decrease of the nematic order parameter (by $18 \%$ for a concentration of $5 \% w t$ ) leads to a significant decrease of almost all nematic properties, in particular to a decrease of the splay elastic constant by $51 \%$. These values in presence of magnetic NPs however reveal no specific enhancement of the dilution effect for active NPs. The nematic order parameter decrease in presence of magnetic NPs suggests that the magnetic moments of the magnetic NPs are not aligned parallel to the average nematic director. This shows an only weak coupling between magnetic moments of NPs and 5CB nematic order parameter in contrast with ferronematics made of large magnetic platelets. This may be due to the spherical and small size of the magnetic NPs. Finally, the magnetic NPs appear to affect more than expected through the combination of dilution effect and order parameter decrease the diffusion coefficient (increasing by $43 \%$ ) and the dielectric anisotropy (decreasing by $43 \%$ ). This confirms 
that a significant structural modification of the LC occurs in presence of magnetic NPs in relation with long range magnetic interactions.

Acknowledgements, avoiding identifying any of the authors prior to peer review 


\section{References:}

[1] K. K. Vardanyana, D. M. Sitaa, R. D. Waltona, I. S. Gurfinkiela, and W. M. Saidel, Liquid crystalline cyanobiphenyl homologues doped with gold nanoparticles, Liq. Cryst. 39, 1083 (2012).

[2] C. J. Hsu, L. J. Lin, M. K. Huang, and C. Y. Huang, Electro-optical effect of gold nanoparticle dispersed in nematic liquid crystals, Crystals. 7, 287 (2017).

[3] U. B. Singh, R. Dhar, R. Dabrowski, and M. B. Pandey, Influence of low concentration silver nanoparticles on the electrical and electro-optical parameters of nematic liquid crystals, Liq. Cryst. 40, 774 (2013).

[4] A. Mouhli, H. Ayeb, T. Othman, J. Fresnais, V. Dupuis, I. R. Nemitz, J. S. Pendery, C. Rosenblatt, O. Sandre, and E. Lacaze, Influence of a dispersion of magnetic and nonmagnetic nanoparticles on the magnetic Fredericksz transition of the liquid crystal 5CB, Phys. Rev. E. 96, 012706 (2017).

[5] G. Yadav, A. Roy, K. Agrahari, R. Katiyar, V. S. Chandel, and R. Manohar, Influence of $\mathrm{Fe}_{2} \mathrm{O}_{3}$ nanoparticles on the birefringence property of weakly polar nematic liquid crystal, Mol. Cryst. Liq. Cryst. 680, 65 (2019).

[6] I. Dierking, From colloids in liquid crystals to colloidal liquid crystals, Liq. Cryst. 46, 2057 (2019).

[7] E. G. Park, C. W. Oh, and H. G. Park, Improvement of the electro-optical properties of nematic liquid crystals doped with strontium titanate nanoparticles at various doping concentrations, Liq. Cryst. 47, 136 (2020).

[8] P. K. Tripathi, A. K. Misra, S. Manohar, S. K. Gupta, and R. Manohar, Improved dielectric and electro-optical parameters of $\mathrm{ZnO}$ nano-particle $\left(8 \% \mathrm{Cu}^{2+}\right)$ doped nematic liquid crystal, J. Mol. Struct. 1035, 371 (2013).

[9] W. T. Chen, P. S. Chen, and C. Y. Chao, Effect of doped insulating nanoparticles on the electro-optical characteristics of nematic liquid crystals, Jpn. J. Appl. Phys. 48, 015006 (2009).

[10] R. Manohar, S. P. Yadav, A. K. Srivastava, A. K. Misra, K. K. Pandey, P. K. Sharma, and A. C. Pandey, Zinc Oxide $(1 \% \mathrm{Cu})$ nanoparticle in nematic liquid crystal: dielectric and electro-optical study, Jpn. J. Appl. Phys. 48, 101501 (2009).

[11] H. Y. Chen, W. Lee, and N. A. Clark, Faster electro-optical response characteristics of a carbon-nanotube-nematic suspension, Appl. Phys. Lett. 90, 033510 (2007). 
[12] C. W. Lee, and W. P. Shih, Quantification of ion trapping effect of carbon nanomaterials in liquid crystals, Mater. Lett. 64, 466 (2010).

[13] M. Kreuzer, T. Tschudi, W. H. de Jeu, and R. Eidenschink, New liquid crystal display with bistability and selective erasure using scattering in filled nematics, App. Phys. Lett. 62, 1712 (1993).

[14] P. S. Chen, C. C. Huang, Y. W. Liu, and C. Y. Chao, Effect of insulatingnanoparticles addition on ion current and voltage-holding ratio in nematic liquid crystal cells, Appl. Phys. Lett. 90, 211111 (2007).

[15] S. Doke, P. Ganguly, and S. Mahamuni, Improvement in molecular alignment of ferroelectric liquid crystal by $\mathrm{Co}-\mathrm{ZnO} / \mathrm{ZnO}$ core/shell quantum dots, Liq. Cryst. 47, 309 (2020).

[16] W. K. Lee, H. J. Hwang, M. J. Chao, H. G. Park, J. W. Han, S. Song, J. H. Jang, and D. S. Seo, CIS-ZnS quantum dots for self-aligned liquid crystal molecules with superior electro-optic properties, Nanoscale. 5, 193 (2013).

[17] A. Rastogi, G. Pathak, J. Herman, A. Srivastava, and R. Manohar, $\mathrm{Cd}_{1-\mathrm{X}} \mathrm{Zn}_{\mathrm{X}} \mathrm{S} / \mathrm{ZnS}$ core/shell quantum dots in nematic liquid crystals to improve material parameter for better performance of liquid crystal based devices, J. Mol. Liq. 255, 93 (2018).

[18] A. Rastogi, K. Agrahari, G. Pathak, A. Srivastava, J. Herman, and R. Manohar, Study of an interesting physical mechanism of memory effect in nematic liquid crystal dispersed with quantum dots, Liq . Cryst. 46, 725 (2019).

[19] U. B. Singh, M. B. Pandey, R. Dhar, and R. Verma, and S. Kumar, Effect of dispersion of $\mathrm{CdSe}$ quantum dots on phase transition, electrical and electro-optical properties of 4PP4OB, Liq. Cryst. 43, 1075 (2016).

[20] S. J. Shivaraja, R. K. Gupta, S. Kumar, and V. Manjuladevi, Effect of functionalised silver nanoparticle on the elastic constants and ionic transport of a nematic liquid crystal, Liq. Cryst. 46, 1868 (2019).

[21] H. G. Walton, Influence of $\mathrm{TiO}_{2}$ nanoparticle doping on the splay and bend elastic constants of the nematic liquid crystal 4'-butyl-4-heptyl-bicyclohexyl-4carbononitrile, CCN47, Mol. Cryst. Liq. Cryst. 574, 60 (2013).

[22] K. Koch, M. Kundt, A. Eremin, H. Nadasi, and A. M. Schmidt, Efficient ferronematic coupling with polymer-brush particles, Phys. Chem. Chem. Phys. 22, 2087 (2020). 
[23] M. V. Rasna, L. Cemok, D. R. Evans, A. Mertelj, and S. Dhara, Phase transitions, optical, dielectric and viscoelastic properties of colloidal suspensions of $\mathrm{BaTiO}_{3}$ nanoparticles and cyanobiphenyl liquid crystals, Liq. Cryst. 42, 1059 (2015).

[24] F. Li, J. West, A. Glushchenko, C. I. Cheon, and Y. Reznikov, Ferroelectric nanoparticle/liquid- crystal colloids for display applications, J. Soc. Inf. Disp. 14, 523 (2006).

[25] N. Podoliak, O. Buchnev, M. Herrington, E. Mavrona, M. Kaczmarek, A. G. Kanaras, E. Stratakis, J. F. Blach, J. F. Henninotd, and M. Warenghemd, Elastic constants, viscosity and response time in nematic liquid crystals doped with ferroelectric nanoparticles, RSC Adv. 4, 46068 (2014).

[26] K. K. Vardanyan, A. Daykin, and B. Kilmer, Study on cyanobiphenyl nematic doped by silver nanoparticles, Liq. Cryst. 44, 1240 (2017).

[27] G. Yadav, G. Pathak, K. Agrahari, M. Kumar, M. S. Khan, V. S. Chandel, and R. Manohar, Improved dielectric and electro-optical parameters of nematic liquid crystal doped with magnetic nanoparticles, Chin. Phys. B 28, 034209 (2019).

[28] R. Katiyar, G. Pathak, A. Srivastava, J. Herman and R. Manohar, Analysis of electro-optical and dielectric parameters of $\mathrm{TiO}_{2}$ nanoparticles dispersed nematic liquid crystal, Soft Materials 16, 126 (2018).

[29] N. Podoliak, O. Buchnev, O. Buluy, G. D’Alesasandro, M Kaczmarek, Y. Reznikov, and T. J. Sluckin, Macroscopic optical effects in low concentration ferronematics, Soft Matter 7, 4742 (2011).

[30] M. F. Prodanov, O. G. Buluy, E. V. Popova, S. A. Gamzaeva, Y. O. Reznikov, and V. V. Vashchenko, Magnetic actuation of a thermodynamically stable colloid of ferromagnetic nanoparticles in a liquid crystal, Soft Matter 12, 6601 (2016).

[31] I. Appel, P. Sharma, and S. D. Wetmore, Structural and electronic properties of barbituric acid and melamine-containing ribonucleosides as plausible components of prebiotic RNA: implications for prebiotic self-assembly, Phys. Chem. Chem. Phys. 19, 12127 (2017).

[32] P. Kopčanský, I. Potočová, M. Timko, M. Koneracká, A.M.G. Jansen, J. Jadzyn, and G. Czechowski, The structural transitions in ferronematics in combined electric and magnetic fields, J. Magn. Magn. Mater, 272-276, 2355 (2004).

[33] P. Kopčanský, N. Tomašovičová, M. Koneracká, V. Závišová, M. Timko, A. Džarová, and A. Šprincová, Structural changes in the 6CHBT liquid crystal doped 
with spherical, rodlike, and chainlike magnetic particles, Phys. Rev. E 78, 011702 (2008).

[34] D. A. Petrov, Molecular-statistical theory of ferromagnetic liquid crystal suspensions, Phys. Rev. E 101, 030701(R) (2020).

[35] A. Mertelj, and D. Lisjak, Ferromagnetic nematic liquid crystals, Liq. Cryst. Rev. 5, 1 (2017).

[36] A. Bogi, and S. Faetti, Elastic, dielectric and optical constants of 4'-pentyl-4cyanobiphenyl, Liq. Cryst. 28, 729 (2001).

[37] C. Maze, Determination of nematic liquid crystal elastic and dielectric properties from the shape of a capacitance-voltage curve, Mol. Cryst. Liq. Cryst. 48, 273 (1978).

[38] M. J. Bradshaw, E. P. Raynes, J. D. Bunning, and T. E. Faber, The Frank constants of some nematic liquid crystals, J. Phys. 46, 1513 (1985).

[39] R. N. Thurston, J. Cheng, R. B. Meyer, and G. D. Boyd, Physical mechanisms of dc switching in a liquid- crystal bistable boundary layer display, J. Appl. Phys. 56, 263 (1984).

[40] M. Cui, and J. R. Kelly, Temperature dependence of visco-elastic properties of 5CB, Mol. Cryst. Liq. Cryst. 331, 49 (1999).

[41] A. G. Chmielewski, Viscosity coefficients of some nematic liquid crystals, Mol. Cryst. Liq. Cryst. 132, 339 (1986).

[42] S. T. Wu, and C. S. Wu, The reorientational behavior of nematic and smectic liquid crystals in a magnetic field, Liq. Cryst. 8, 171 (1990).

[43] K. Skarp, S. T. Lagerwall, B. Stebler, Measurements of hydrodynamic parameters for nematic 5CB, Mol. Cryst. Liq. Cryst. 60, 215 (1980).

[44] P. P. Karat, and N. V. Madhusudana, Elasticity and orientational order in some 4'n-alkyl-4-cyanobiphenyls: Part II, Mol. Cryst. Liq. Cryst. 40, 239 (1977).

[45] S. V. Burylov and A. N. Zakhlevnykh, Magnetically induced bistable behavior of ferronematic liquid crystals, Phys. Rev. E 88, 052503 (2013).

[46] M. V. Gorkunov and M. A. Osipov, Mean-field theory of a nematic liquid crystal doped with anisotropic nanoparticles, Soft Matter 7, 4348 (2011).

[47] Y. L. Raikher, V. I. Stepanov, and A. N. Zakhlevnykh, Hybrid polymer and low molecular weight gels-dynamic two-component soft materials with both responsive and robust nanoscale networks, Soft Matter 9, 177 (2013). 
[48] I. Haller, Thermodynamic and static properties of liquid crystals, Prog. Solid. state Chem. 10, 103 (1975).

[49] B. Kundu, R. Pratibha, and N. V. Madhusudana, Orientational order in liquid crystals exhibited by some binary mixtures of rod-like and bent-core molecules, Eur. Phys. E 31, 145 (2010).

[50] P. Sathyanarayana, V. S. R. Jampani, M. Skarabot, I. Musevic, K. V. Le, H. Takezoe, and S. Dhara, Viscoelasticity of ambient-temperature nematic binary mixtures of bent-core and rodlike molecules, Phys. Rev. E 85, 011702 (2012).

[51] S. Kaur, J. Addis, C. Greco, A. Ferrarini, V. Görtz, J. W. Goodby, and H. F. Gleeson, Understanding the distinctive elastic constants in an oxadiazole bent-core nematic liquid crystal, Phys. Rev. E 86, 041703 (2012).

[52] R. G. Horn, Refractive indices and order parameters of two liquid crystals, Le Journal de Physique. 39, 105 (1978).

[53] I. Abdulhalim, Liquid crystal active nanophotonics and plasmonics: from science to devices, J. Nanophotonics. 6, 061001 (2012).

[54] G. Yadav, M. Kumar, A. Srivastava, and R. Manohar, $\mathrm{SiO}_{2}$ nanoparticles doped nematic liquid crystal system: An experimental investigation on optical and dielectric properties, Chin. J. Phys. 57, 82 (2019).

[55] K. K. Vardanyan, D.M. Sita, R.D. Walton, I.S. Gurfinkiel, and W.M. Saidel, Liquid crystalline cyanobiphenyl homologues doped with gold nanoparticles, Liq. Cryst. 39,1083 (2012).

[56] K. K. Vardanya, R.D. Walto, D.M. Sita, I.S. Gurfinkiel, and W.M. Saidel, Study of pentyl-cyanobiphenyl nematic doped with gold nanoparticles, Liq. Cryst. 39, 595 (2012).

[57] T. Tóth-Katona, P. Salamon, N. Éber, N. Tomašovičová, Z. Mitróová and P. Kopčanský, High concentration ferronematics in low magnetic fields, J. Magn. Magn. Mater, 372, 117 (2014).

[58] O. Buluy, D. Burseva, M. R. Hakobyan, J. W. Goodby, M. A. Kolosov, Yu. Reznikov,R. S. Hakobyan, K. Slyusarenko, M. F. Prodanov and V. Vashchenko, Influence of surface treatment of ferromagnetic nanoparticles on properties of thermotropic nematic liquid crystals, Mol. Cryst. Liq. Cryst, 560, 149 (2012). 
[59] V. Gdovinová, M. A. Schroer, N. Tomašovičová, I. Appel, S. Behrens, J. Majorošová, J. Kováč, D. I. Svergun and P. Kopčanský, Structuralization of magnetic nanoparticles in 5CB liquid crystals, Soft Matter, 13, 7890 (2017).

[60] A. Mertelj, D. Lisjak, M. Drofenik, and M. Čopič, Ferromagnetism in suspensions of magnetic platelets in liquid crystal, Nature 504, 237 (2013).

[61] F. Haraguchi, K. I. Inoue, N. Toshima, S. Kobayashi, and K. Takatoh, Reduction of the threshold voltages of nematic liquid crystal electrooptical devices by doping inorganic nanoparticles, Jpn. J. Appl. Phys. 46, L796 (2007).

[62] V. Gdovinová, N. Tomašovičová, N. Éber, T. Tóth-Katona, V. Závišová, M. Timkoa, and P. Kopčanský, Influence of the anisometry of magnetic particles on the isotropic-nematic phase transition, Liq. Cryst. 41, 1773 (2014).

[63] J. Prost, G. Sigaud, and B. Regaya, On the thermal dependence of the twist viscosity in nematic liquid crystals, J. Phys. Lett. (Paris) 37, 341 (1976).

[64] V. V. Belyaev, S. Ivanov, and M. F. Grebenkin, Temperature dependence of rotational viscosity of nematic liquid crystals, Sov. Phys. Crystallogr. 30, 674 (1985).

[65] A. G. Chmielewski, Viscosity coefficients of some nematic liquid crystals, Mol. Cryst. Liq. Cryst. 132, 339 (1986).

[66] L. Blinov, and V. Chigrinov, Electrooptic Effects in Liquid Crystal Materials (Springer, New York, 1994).

[67] V. Belyaev, Physical properties of liquid crystals: nematics, edited by A. Dunmur, G. Fukuda, and A. Luckhurst (INSPEC, London, 2001), p. 414. 
Table 1. Nematic isotropic phase transition temperatures $T_{N I}$ of different samples.

\begin{tabular}{c|c|c|c|c|c|c|c}
\hline \multicolumn{2}{c|}{ Concentration } & $0 \%$ & $1.5 \%$ & $3.2 \%$ & $5 \%$ & $7.5 \%$ & $10 \%$ \\
\hline \multirow{2}{*}{$T_{N I}\left({ }^{\circ} \mathrm{C}\right)$} & $\gamma \mathrm{Fe}_{2} \mathrm{O}_{3}$ & 35.1 & 33.9 & 33.1 & 29.5 & 32.4 & 32.4 \\
\cline { 2 - 8 } & $\mathrm{CeO}_{2}$ & 35.1 & 34.0 & 33.6 & 33.8 & 33.7 & 32.8 \\
\hline
\end{tabular}




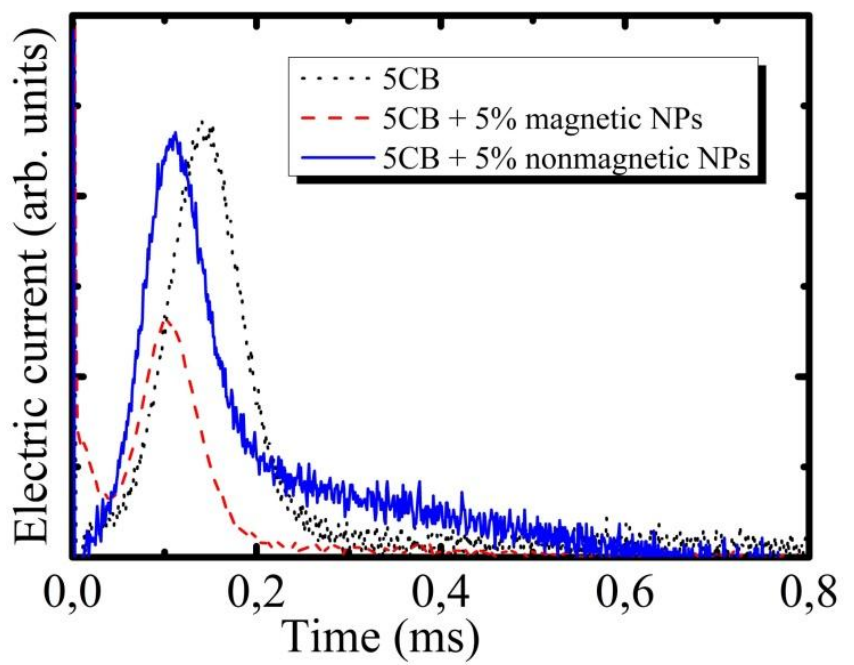

FIG. 1. (Color online) Transient currents induced by a DC voltage of $14 \mathrm{~V}$ applied to a cell filled with pure 5CB nematic liquid crystal (black dotted curve) and two cells filled with $5 \mathrm{CB}$ doped with $5 \%$ of magnetic NPs (red dashed curve) and $5 \%$ of nonmagnetic NPs (blue solid curve) at constant temperature of $27^{\circ} \mathrm{C}$.

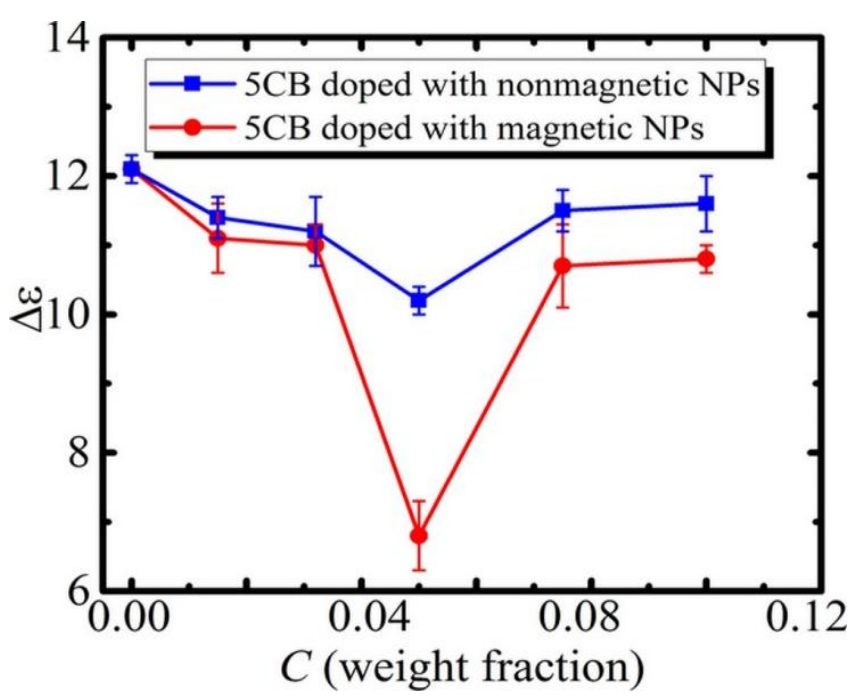

FIG.2. (Color online) Variation of the dielectric anisotropy as function of the nanoparticle weight concentration for nonmagnetic (blue square) and magnetic (red circle) nanoparticles. 


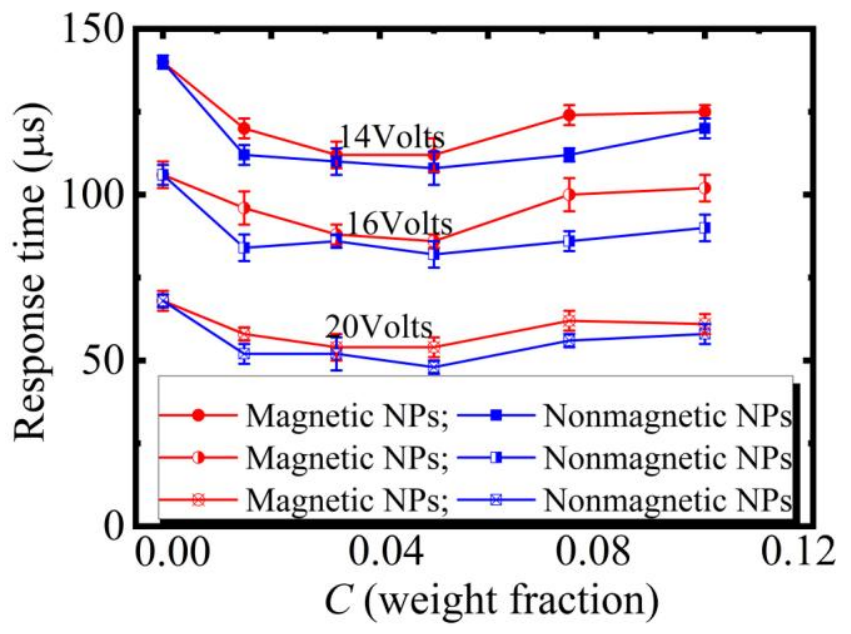

FIG.3. (Color online) Evolution of the response time as a function of the nanoparticle weight fraction concentration for nonmagnetic (blue square) and magnetic (red circle) nanoparticles.

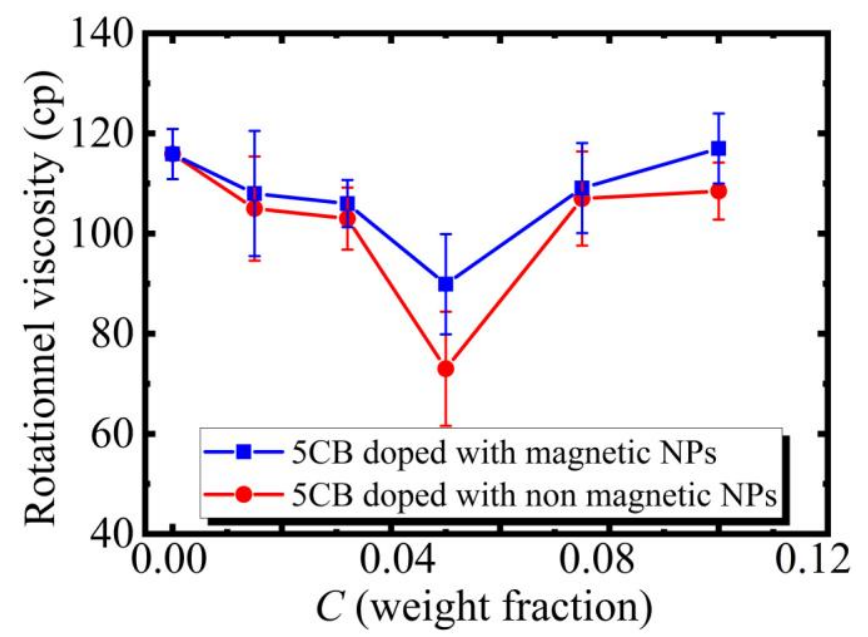

FIG.4. (Color online) Variation of rotational viscosity as a function of the nanoparticle weight concentration for nonmagnetic (blue square) and magnetic (red circle) nanoparticles. 


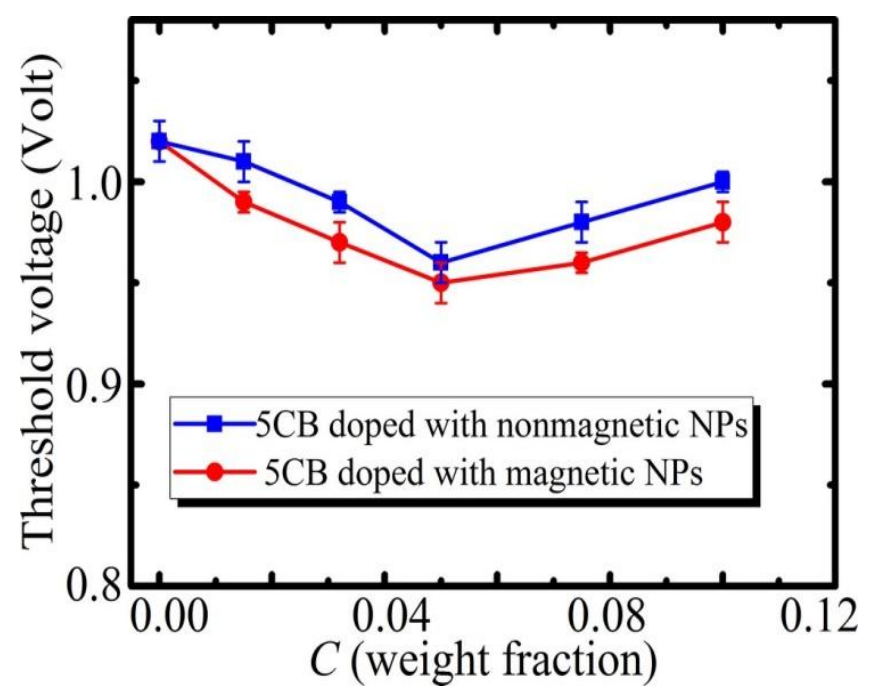

FIG.5. (Color online) Variation of the threshold voltage as a function of the nanoparticle weight concentration for nonmagnetic (blue square) and magnetic (red circle) nanoparticles.

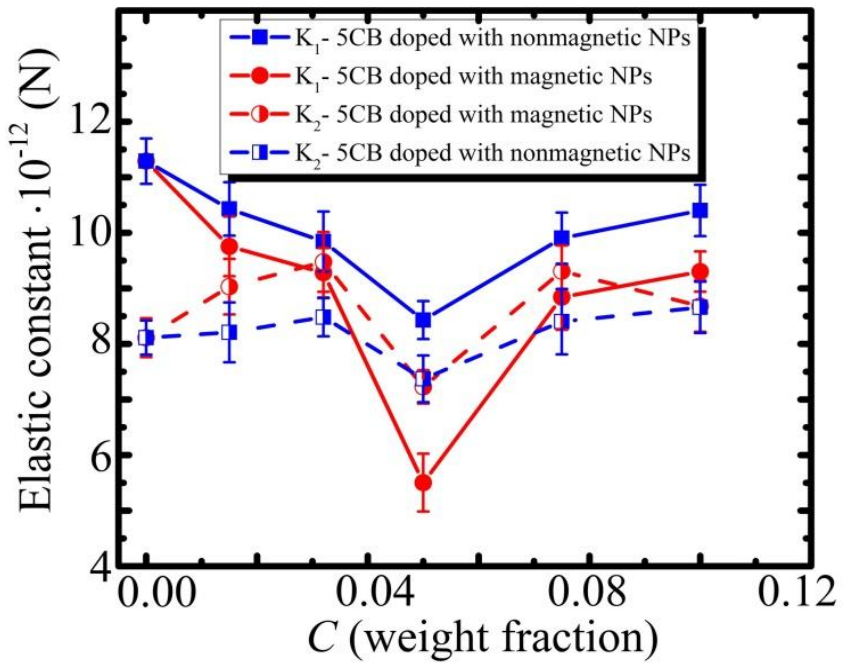

FIG.6. (Color online) Variation of the splay (solid line) and twist (dash line) elastic constants as a function of the nanoparticle weight concentration for nonmagnetic (blue square) and magnetic nanoparticles (red circle). 


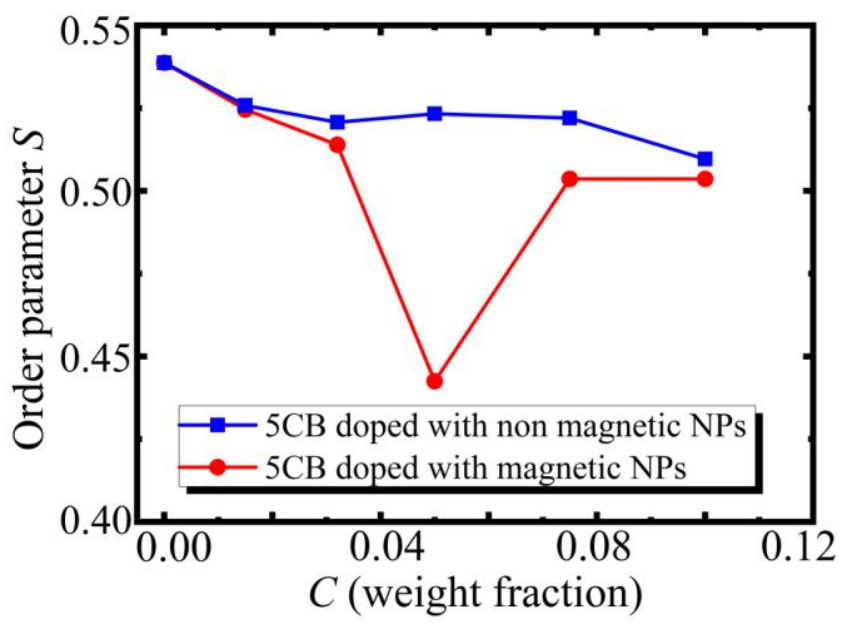

FIG.7. (Color online) Evolution of the order parameter $S$ as a function of the nanoparticle concentration for nonmagnetic (blue square) and magnetic (red circle) nanoparticles.

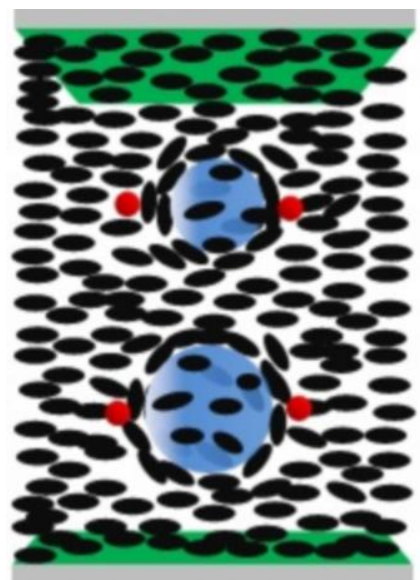

(a)

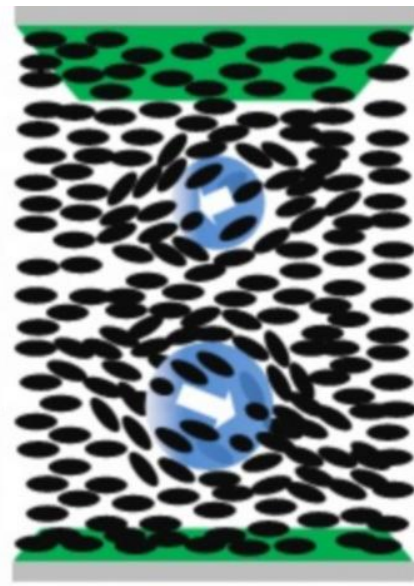

(b)

FIG.8. (Color online) Scheme illustrates the expected nematic molecules orientation around the nonmagnetic NPs (a) and magnetic NPs (b). The red dot is indicating the expected position of the defect in presence of nonmagnetic NPs. The arrow represents the magnetic moment of the NPs which should orient locally the molecules parallel to its direction. 